\title{
Testing magnetically confined wind shock models for $\beta$ Cephei using XMM-Newton and Chandra phase-resolved X-ray observations
}

\author{
F. Favata ${ }^{1}$, C. Neiner ${ }^{2}$, P. Testa ${ }^{3}$, G. Hussain ${ }^{4}$, and J. Sanz-Forcada ${ }^{5}$ \\ ${ }^{1}$ European Space Agency, 8-10 rue Mario Nikis, 75015 Paris, France \\ e-mail: Fabio.Favata@esa.int \\ 2 GEPI, UMR 8111 du CNRS, 5 place Jules Janssen, 92195 Meudon Cedex, France \\ 3 Kavlis Institute for Astrophysics and Space Research, MIT, Cambridge, Mass., USA \\ ${ }^{4}$ European Southern Observatory, Garching bei München, Germany \\ 5 Laboratorio de Astrofísica Espacial y Física Fundamental, INTA, PO Box 50727, 28080 Madrid, Spain
}

Received 22 August 2007 / Accepted 4 June 2008

\section{ABSTRACT}

\begin{abstract}
Aims. We have performed a set of high- and low-spectral resolution phase-resolved X-ray observations of the magnetic B star $\beta$ Cep, for which theoretical models predict the presence of a confined wind emitting X-rays from stationary shocks. Given the peculiar geometry of $\beta \mathrm{Cep}$, some of the models predict strong rotational modulation of the X-ray emission, while other models predict a much lower amplitude modulation at 90 deg phase shift from the modulation predicted from the first group of models. Our observations were designed to provide a stringent test of such models.

Methods. We obtained four observations spaced in rotational phase with XMM-Newton (using both the EPIC cameras and the RGS spectrograph) and with Chandra (using the LETG spectrograph). A detailed analysis of the data was performed to derive both photometric and spectral parameters from the EPIC data, searching for rotational modulation, and to derive the location of the X-ray plasma from the line ratios in the He-like triplets of $\mathrm{N}, \mathrm{O}$, and Ne from the RGS data. The LETG data were used to constrain the presence of bulk motions in the plasma.

Results. The strong rotational modulation predicted by the early, static magnetically-confined wind model for the X-ray emission is not observed in $\beta$ Cep. The small modulation present goes in the opposite direction, pointing to the absence of any optically thick disk of neutral material, and showing a modulation consistent with the later, dynamic models of magnetically-confined wind models in B stars. The lack of observed bulk motion points to the plasma being confined by a magnetic field, but the low plasma temperature and lack of any flaring show that the plasma is not heated by magnetic reconnection. Therefore, the observations point to X-ray emission from shocks in a magnetically confined wind, with no evidence of an optically thick, dense disk at the magnetic equator.
\end{abstract}

Key words. stars: emission-line, Be $-\mathrm{X}$-rays: stars

\section{Introduction}

Early-type stars were established as strong soft X-ray sources during the first survey of stellar X-ray emission conducted with the Einstein observatory (Vaiana et al. 1981). O and B stars lack the external convection layer which is an essential component of the dynamo in late-type dwarfs, and thus are expected to lack the highly structured magnetic fields that confine and heat solartype coronae. The observed X-ray emission is of thermal nature, and in general of lower temperature than observed in active late-type stars. The mechanism proposed initially to explain the observed X-ray emission was self shocking in the radiativelydriven fast, strong stellar winds which are a characteristic of massive stars (Lucy \& White 1980). This scenario makes a welldefined prediction, i.e. that emission lines should be broad and blue-shifted. High spectral resolution observations, made possible by the launch of XMM-Newton and Chandra, have shown that some massive stars show the expected signature of X-ray emission from the wind (e.g. $\zeta$ Pup, Cassinelli et al. 2001). Other stars, however, show narrow spectral lines (e.g. $\theta^{1}$ Ori C, Gagné et al. 2005). Narrow lines imply a low-velocity plasma that has been interpreted as a confined plasma, likely requiring magnetic fields (e.g. Schulz et al. 2003; Schulz et al. 2006, and reference therein). Alternative scenarios, which do not require magnetic confinement for the X-ray emission from early-type stars showing narrow lines, have also been proposed (Cohen et al. 2006; Leutenegger et al. 2006). A survey of early-type high-resolution spectra was recently performed by Waldron \& Cassinelli (2007).

Dipolar fields have recently been detected in a number of massive stars, in some cases with the magnetic axis at a significant angle from the rotation axis. Scenarios that imply magnetic confinement of the wind by a dipolar magnetic field have been developed, and (as discussed below) succeed in explaining several of the phenomena observed in massive stars with measured magnetic fields and X-ray emission. For example, Babel \& Montmerle (1997a) developed a magnetically-confined wind shock model (MCWS) to explain the characteristics of the X-ray emission from Ap and Bp stars, and Babel \& Montmerle (1997b) applied the MCWS model to explain some of the salient characteristics of the X-ray emission of $\theta^{1}$ Ori C, one of only two O-type stars with a detected magnetic field. Strong rotational modulation is present in the X-ray emission from $\theta^{1}$ Ori $\mathrm{C}$, with a nearly sinusoidal light curve and a $\simeq 50 \%$ peak-to-peak modulation amplitude (Gagné et al. 1997; Stelzer et al. 2005); 
such strong modulation agrees well with the predictions of the MCWS scenario. Interestingly, another $\mathrm{O}$ star for which a magnetic field was recently detected, HD 191612 (Donati et al. 2006) has an unusually slow rotation rate, and it also has a field with a strong dipolar component. The latest discovery of a magnetic O star ( $\zeta$ Ori A, Bouret et al. 2008) concerns a more complex magnetic topology.

As discussed in Sect. 2, $\beta$ Cep has a significant dipolar magnetic field $(\simeq 360 \mathrm{G}$, Henrichs et al. 2000), with the magnetic axis at almost 90 deg from the rotational axis, and is therefore a particularly interesting star for the study of the magnetically confined wind model. Its X-ray emission was detected by the Einstein observatory. Donati et al. (2001) (hereafter D01) devised a detailed model for these and the later ROSAT observations, based on the MCWS scenario of Babel \& Montmerle (1997b). They presented clear and verifiable predictions about the temporal variability of the X-ray emission and on its spectrum, as well as on the spatial location of the X-ray plasma. Later work based on dynamical modeling of the wind-magnetic-field interaction, however, indicated that the thick disk predicted by Babel \& Montmerle (1997b) would be unlikely to form around a star such as $\beta$ Cep (Gagné et al. 2005; ud-Doula \& Owocki 2002; Townsend \& Owocki 2005).

We have completed a campaign of X-ray observations using both Chandra and XMM-Newton, which designed to test the MCWS-based model of the X-ray emission of $\beta$ Cep. The observations were designed to determine whether the X-ray emission does indeed vary with rotational phase as predicted by the D01 model and (by using triplet ratios and Doppler shifts) at which distance from the stellar photosphere the bulk of the X-ray emission is concentrated. We were interested in observing $\beta$ Cep in X-ray because it was considered a Be star, although, as discussed in Sect. 2, recent observations have suggested that the Be phenomenology is due to the secondary (presumably much less $\mathrm{X}$-ray active) companion in the $\beta$ Cep system, rather than to the magnetic, $\mathrm{X}$-ray active primary.

The present paper is structured as follows: after the Introduction, the characteristics of our target star, $\beta$ Cep, are discussed in Sect. 2. XMM-Newton and Chandra observations are discussed in Sect. 3 with their analysis, and the corresponding results are presented in Sect. 4. Finally, we present our conclusions in Sect. 5.

\section{The star $\beta$ Cep}

Stars in the $\beta$ Cep class are early-B subgiants or giants that exhibit coherent short-period radial velocity variations, successfully explained in terms of pulsations with periods ranging from about 3 to $8 \mathrm{~h}$. The driving mechanism of pulsation in $\beta$ Cep stars is the $\kappa$ mechanism, i.e. an effect of the changing opacity of iron-peak elements deep in the stellar envelope (Dziembowski \& Pamiatnykh 1993).

The prototype of this class, the star $\beta$ Cep itself (HD 205021 , HR 8238), long classified as a B1IIIe star, is at a Hipparcos distance of $182 \mathrm{pc}$. The literature values of the basic photospheric parameters of $\beta$ Cep span a relatively wide range: $T_{\mathrm{eff}}$ varies from $24000 \mathrm{~K}$ (e.g. Heynderickx et al. 1994) to $27000 \mathrm{~K}$ (e.g. Tian et al. 2003), $\log g$ from 3.31 (e.g. Heynderickx et al. 1994) to 4.07 (e.g. Cugier \& Boratyn 1992), while $M$ ranges from $9.9 M_{\odot}$ (D01) to $16.4 M_{\odot}$ (Heynderickx et al. 1994). The radius $R$ is estimated to be between $6.5 R_{\odot}$ (D01) and $8.6 R_{\odot}$ (Hutchings \& Hill 1977). The main pulsation period of $\beta \mathrm{Cep}, 4 \mathrm{~h}$ and $34 \mathrm{~min}$, corresponds to a radial $p$ mode, but additional periods exist due to non-radial modes. While
Table 1. The photospheric abundances of $\beta$ Cep as determined by Morel et al. (2006). The first column gives the ratio between the photospheric abundance of $\beta$ Cep and the standard photospheric abundance of the Sun of Grevesse \& Sauval (1998). The second column indicates the abundance relative to the solar abundances determined using 3D calculations by Asplund et al. (2005). The relative $1 \sigma$ errors are given in Col. 3 .

\begin{tabular}{rccc}
\hline \hline & $M / M_{\odot 1 D}$ & $M / M_{\odot 3 D}$ & $1 \sigma(\mathrm{dex})$ \\
\hline $\mathrm{C}$ & 0.32 & 0.43 & 0.10 \\
$\mathrm{~N}$ & 0.98 & 1.35 & 0.13 \\
$\mathrm{O}$ & 0.44 & 0.65 & 0.14 \\
$\mathrm{Mg}$ & 0.54 & 0.60 & 0.21 \\
$\mathrm{Al}$ & 0.36 & 0.45 & 0.16 \\
$\mathrm{Si}$ & 0.36 & 0.40 & 0.23 \\
$\mathrm{~S}$ & 0.65 & 1.00 & 0.37 \\
$\mathrm{Fe}$ & 0.55 & 0.62 & 0.23 \\
\hline
\end{tabular}

Daszyńska-Daszkiewicz \& Niemczura (2005) and Niemczura \& Daszyńska-Daszkiewicz (2005) derive a photospheric metal abundance close to solar $(m / H=-0.07 \pm 0.10)$ from the analysis of IUE spectra, Morel et al. (2006) performed a detailed abundance analysis of the photosphere of $\beta$ Cep based on high-resolution optical spectra, deriving abundances significantly lower than solar values, except for $\mathrm{N}$ and possibly $\mathrm{S}$, as detailed in Table 1. In the following, we adopt the abundances of Morel et al. (2006).

$\beta$ Cep is the primary star of a triple system. The visual companion, ADS $15032 \mathrm{~B}$, is a $V=7.9 \mathrm{~A} 2.5 \mathrm{~V}$ star located at 13.4 arcsec from the primary. It has an orbital period of about $91.6 \mathrm{yr}$ and was at periastron in 2006 (Pigulski \& Boratyn 1992). The third component of the system, a physical companion, is a $V=6.6 \mathrm{~B} 6-8$ star situated at 0.1 arcsec from the primary (Schnerr et al. 2006), detected by means of speckle interferometry (Gezari et al. 1972).

The $\beta$ Cep system also exhibits emission in its Balmer lines, so that the primary itself has long been considered to be a Be star. In Be star, the Balmer emission is due to the presence of circumstellar matter ejected by the star. The Be character is usually variable with time, and the $\beta$ Cep system is no exception. The time evolution of the intensity of its $\mathrm{H} \alpha$ emission line is shown in Fig. 1: major outbursts occurred around 1990 and 2001. At the time of our X-ray observations in 2005 (see Fig. 1), the $\mathrm{H} \alpha$ emission was decreasing but still visible in the line profile. However, Be stars usually rotate fast, at about $v \sin i=250 \mathrm{~km} \mathrm{~s}^{-1}$ on average, while the $\beta$ Cep primary rotates intrinsically slowly, at $v \sin i=20 \mathrm{~km} \mathrm{~s}^{-1}$, and $i=60 \mathrm{deg}$ (Abt et al. 2002), which implies that it is different from classical Be stars.

The condundrum of the unusually low rotation speed of $\beta$ Cep relative to the values typical for Be stars was apparently resolved by Schnerr et al. (2006), who argued that the $\mathrm{H} \alpha$ emission does not originate in the bright primary, but rather in the fainter speckle companion. This implies that the $\beta$ Cep primary is a normal B star, rather than a peculiar Be star.

The rotational period of $\beta$ Cep is almost exactly 12 days, and in the present study we use the ephemeris of D01,

$\mathrm{JD}=2451238.15+N \times 12.00092$

where phase 0 is the phase at which the longitudinal magnetic field (see below) is at a maximum. This ephemeris is consistent, within the error bars, with the values published by Henrichs et al. (2000) and Henrichs et al. (2005). 


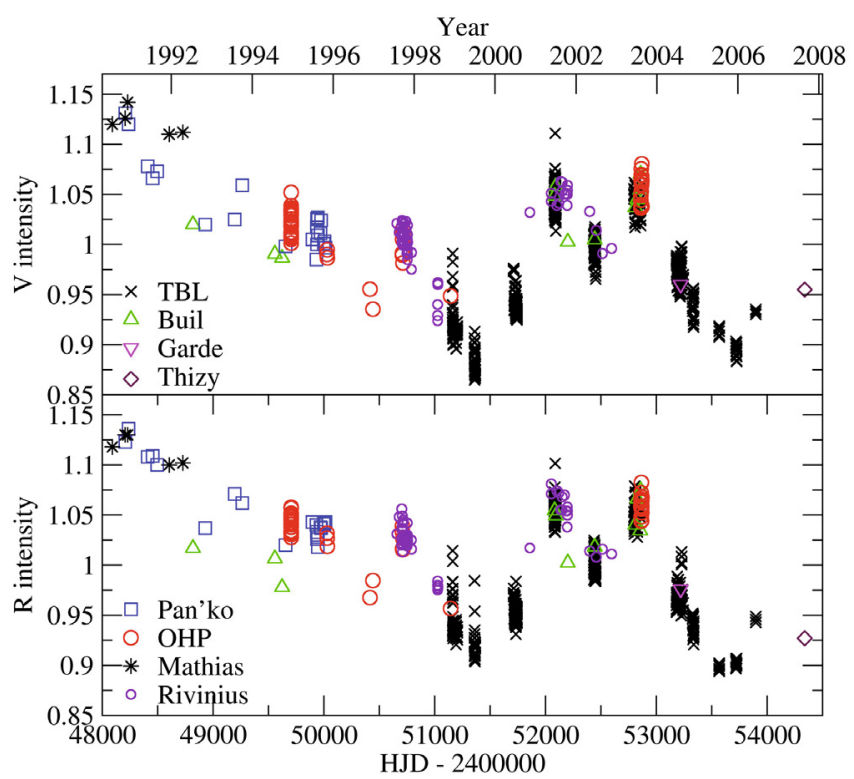

Fig. 1. Evolution of the intensity of the $\mathrm{H} \alpha$ emission of $\beta$ Cep with time. Data were obtained at the Telescope Bernard Lyot (TBL) of Pic du Midi Observatory and Observatoire de Haute-Provence (OHP), and collected from the literature (Pan'ko \& Tarasov 1997), by amateurs astronomers (Buil, Garde, Thizy) and private communications (Mathias, Rivinius).

\subsection{The magnetic field of $\beta$ Cep}

Magnetic fields have been detected in an increasing number of normal massive stars, in addition to the well known fields of $\mathrm{Ap} / \mathrm{Bp}$ stars. In particular, magnetic fields have been discovered in a few $\beta$ Cep stars, including $\beta$ Cep itself (Henrichs et al. 2000). The longitudinal magnetic field of $\beta$ Cep is observed to vary with the 12 day rotation period.

These observations of magnetic massive stars can usually be explained by the magnetic oblique rotator model (Stibbs 1950). In this model, the magnetic field structure is not symmetric about the rotation axis of the star. For the simplest case of a dipole field, this implies that the axis of the dipole and the axis of rotation of the star do not coincide. The observed geometrical configuration can then be characterized by the inclination angle $i$ between the observer's line-of-sight and the stellar rotation axis, and by the obliquity angle $\beta$ between the magnetic axis and the rotation axis. In the case of a dipolar magnetic star, as the star rotates, the aspect of its visible hemisphere changes. This leads to variations in a number of observables with the stellar rotation period. $\beta$ Cep hosts such a magnetic oblique dipole field with an inclination angle $i=60^{\circ}$ and its rotation and magnetic axes are almost perpendicular to each other $\left(\beta=85^{\circ}\right)$. Therefore, as the star rotates, the observer successively sees the magnetic equator nearly edge-on or face-on. Figure 2 shows the magnetic oblique dipole model of $\beta$ Cep at different rotation phases, with the magnetic equator drawn as a thicker black line.

In the presence of a sufficiently strong dipolar magnetic field, the stellar wind escapes from the star via its magnetic poles and follows the magnetic field lines. As the star rotates one thus observes a variation in the equivalent width of the windsensitive lines such as the UV resonance lines of highly ionized species. Such variations were observed for $\beta$ Cep by Henrichs et al. (2000), who found that the C IV line is strongly modulated with the rotation period of $12 \mathrm{~d}$, maintaining phase coherence over several years. This shows that the transition-region temperature material responsible for C IV emission is magnetically

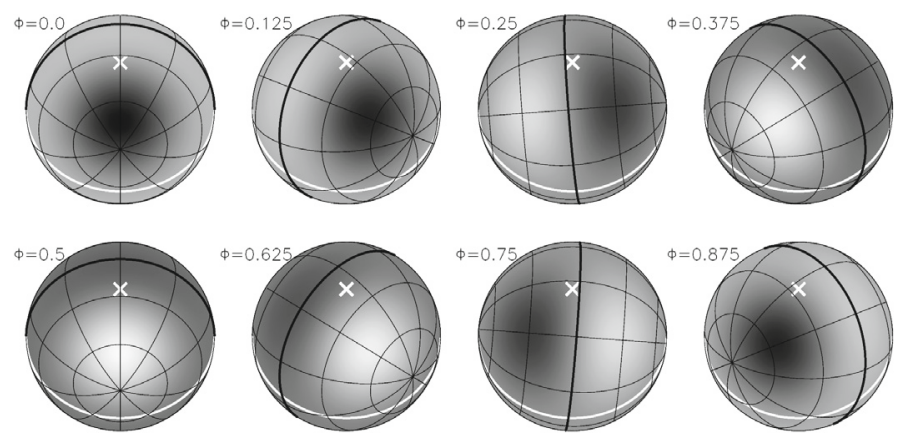

Fig. 2. Greyscale representations of the relative contribution of the magnetic dipole to the integrated longitudinal magnetic field in the visible hemisphere of $\beta \mathrm{Cep}$, at different rotational phases, with step $\delta \Phi=0.125$. Phase 0 is the phase at which the longitudinal field is at maximum, as in the ephemeris above. The black color corresponds to positive field values and the white color to negative field values. A grid of magnetic longitudes and latitudes is overplotted, with the magnetic equator shown as a thicker black line. The rotation axis and equator are indicated by a white cross and line respectively. Although the strongest magnetic field is at the magnetic poles, the positions on the stellar surface that contribute the most to the longitudinal field are not at the poles, due to a geometrical effect and the limb darkening effect.

confined (and, incidentally, that it is associated with the primary, rather than the Be secondary).

If the magnetic field is sufficiently strong the wind can be fully magnetically confined in a region close to the star (Babel \& Montmerle 1997b). In this case, the stellar wind particles originating from both magnetic poles, forced to follow the magnetic field lines, collide at the magnetic equator creating a decretion disk or circumstellar clouds. The MCWS model was successfully applied to explain the characteristics of the X-ray emission in a number of massive stars, in particular $\theta^{1}$ Ori $C$ (Donati et al. 2002; Gagné et al. 2005). D01 showed that the MCWS appears also to apply to $\beta \mathrm{Cep}$, explaining at the same time the rotation modulation observed in the wind-sensitive UV lines and in the longitudinal field measurements.

\subsection{The $X$-ray emission of $\beta$ Cep}

As mentioned above, stars hotter than B4 have sufficiently strong winds to produce X-rays in wind shocks. However, in the case of magnetically confined winds, shocks may also take place at the stellar equator, where the winds originating in the two magnetic poles collide. The resulting shock is expected to produce X-ray emission localized at or close to the magnetic equator.

$\beta$ Cep has been known to be an X-ray source since its observation with Einstein. The X-ray spectrum, derived from ROSAT All Sky Survey observations obtained in 1990 and reported by Berghoefer et al. (1996), was interpreted as originating in a thermal plasma with an X-ray temperature of $0.24 \mathrm{keV}$ and an X-ray luminosity of $6.5 \times 10^{29} \mathrm{erg} \mathrm{s}^{-1}$, for an assumed distance of $71 \mathrm{pc}$ (or $L_{\mathrm{X}}=4.2 \times 10^{30} \mathrm{erg} \mathrm{s}^{-1}$ rescaled to the Hipparcos distance of $182 \mathrm{pc})$. Agrawal et al. (1984) derived $L_{X}=9.1 \times 10^{30} \mathrm{erg} \mathrm{s}^{-1}$ from Einstein IPC observations, assuming a distance of $263 \mathrm{pc}$, or $L_{\mathrm{X}}=4.3 \times 10^{30} \mathrm{erg} \mathrm{s}^{-1}$ rescaled to the Hipparcos distance, very close to the value derived above for ROSAT data. Cohen (2000) claimed the detection of a 7\% modulation in the X-ray emission of $\beta$ Cep at the $4.57 \mathrm{~h}$ pulsation period, based on analysis of ROSAT All Sky Survey data.

The MCWS model by Babel \& Montmerle (1997b) makes clear predictions about the characteristics of the X-ray 
Table 2. The spectral parameters derived from the XMM-Newton EPIC pn observations, with the relative phase coverage. The reported flux is measured in a $0.3-7.0 \mathrm{keV}$ band.

\begin{tabular}{lrrrr}
\hline \hline Obs. & 201 & 301 & 401 & 501 \\
\hline Start date & $2005-07-27$ & $2005-07-29$ & $2005-08-02$ & $2005-08-06$ \\
Start time (UT) & $04: 07: 27$ & $01: 36: 19$ & $05: 53: 00$ & $05: 10: 42$ \\
Obs. duration (s) & 39234 & 41030 & 42504 & 38170 \\
$\phi$ begin & 0.03 & 0.19 & 0.54 & 0.87 \\
$\phi$ end & 0.07 & 0.23 & 0.58 & 0.91 \\
$N(\mathrm{H})\left(10^{20} \mathrm{~cm}^{-2}\right)$ & $4.5 \pm 0.1$ & $5.2 \pm 0.2$ & $3.2 \pm 0.1$ & $5.8 \pm 0.2$ \\
$T_{1}(\mathrm{keV})$ & $0.23 \pm 0.06$ & $0.24 \pm 0.01$ & $0.29 \pm 0.01$ & $0.24 \pm 0.01$ \\
$T_{2}(\mathrm{keV})$ & $0.69 \pm 0.03$ & $1.14 \pm 0.1$ & $2.0 \pm 1.4$ & $0.63 \pm 0.02$ \\
$E M_{1}\left(10^{53} \mathrm{~cm}^{-3}\right)$ & $12.0 \pm 5.0$ & $18.8 \pm 6.4$ & $16.0 \pm 4.2$ & $17.2 \pm 6.4$ \\
$E M_{2}\left(10^{53} \mathrm{~cm}^{-3}\right)$ & $0.96 \pm 0.42$ & $0.45 \pm 0.12$ & $0.14 \pm 0.20$ & $0.83 \pm 0.42$ \\
$\mathrm{llux}_{\left(10^{-12} \mathrm{erg} \mathrm{cm}^{-2} \mathrm{~s}^{-1}\right)}$ & 1.01 & 0.89 & 0.99 & 0.92 \\
$\mathrm{O} / \mathrm{O}_{\odot}$ & $0.13 \pm 0.03$ & $0.08 \pm 0.02$ & $0.08 \pm 0.02$ & $0.09 \pm 0.02$ \\
$\mathrm{Ne} / \mathrm{Ne} \mathrm{S}_{\odot}$ & $0.18 \pm 0.05$ & $0.22 \pm 0.06$ & $0.16 \pm 0.03$ & $0.16 \pm 0.03$ \\
$\mathrm{Si} / \mathrm{Si} i_{\odot}$ & $0.72 \pm 0.24$ & $0.23 \pm 0.13$ & $0.40 \pm 0.13$ & $0.41 \pm 0.16$ \\
$\mathrm{Fe} / \mathrm{Fe}_{\odot}$ & $0.45 \pm 0.13$ & $0.28 \pm 0.08$ & $0.17 \pm 0.03$ & $0.32 \pm 0.08$ \\
\hline
\end{tabular}

emission from $\beta$ Cep, as extensively discussed in Sect. 4.2 of D01. The luminosity, temperature distribution, and emission measure predicted by the plasma distribution of this model are, in the $0.1-2.0 \mathrm{keV}$ range of the ROSAT PSPC, $7.2 \times 10^{30} \mathrm{erg} \mathrm{s}^{-1}$, $3.2 \mathrm{MK}$, and $4.5 \times 10^{53} \mathrm{~cm}^{-3}$ for the X-ray luminosity, temperature, and emission measure, respectively. These are in good agreement with the values obtained for the ROSAT observation (Berghoefer et al. 1996).

The same model makes a testable prediction regarding the temporal variability of the X-ray emission, i.e. that is should show which strong modulation as a function of rotational phase. The X-ray emission is produced in shocks located on both sides of the cooling disk, whose density is sufficiently high to ensure that it is completely opaque to the X-rays emitted by the shock. As a consequence, when the system is viewed with the disk edge-on, X-rays reach the observer from both shocks (on the magnetic north and south side of the disk), whereas when the disk is seen face-on, only one of the shocks is visible in X-rays. The expected X-ray modulation amplitude in the MCWS scenario of D01 is thus of the order of 50\%, although (as discussed by D01) the predicted shape of the X-ray light curve can be strongly affected by the characteristics of the disk, for example its warp.

The dynamical models of ud-Doula \& Owocki (2002) and Gagné et al. (2005), when applied to $\beta$ Cep, make different predictions for the modulation of its X-ray emission: in particular, the magnetic confinement parameter

$\eta_{*}=\frac{B_{*}^{2}(\pi / 2) R_{*}^{2}}{\dot{M} v_{\infty}}$

using the appropriate values for $\beta \operatorname{Cep}\left(B=360 \mathrm{G}, R_{*}=6.5 R_{\odot}\right.$, $\dot{M}=10^{-9} M_{\odot} \mathrm{yr}^{-1}, v_{\infty}=800-1500 \mathrm{~km} \mathrm{~s}^{-1}$ ) would be high $\left(\eta_{*} \simeq 1000\right)$, so that the Gagné et al. (2005) model predicts that the magnetosphere should be rigid out to the Alfven radius $\left(R_{\mathrm{A}} \simeq 5 R_{*}\right)$ and the $\mathrm{X}$-ray shocks form just outside the Alfven radius but inside the Kepler radius $R_{\mathrm{K}}=7 R_{*}$ (Gagne, private communication). This implies a small modulation in the $\mathrm{X}$-ray activity, at the $\simeq 5 \%$ level, with a minimum that occurs when the star is seen with the magnetic equator edge-on, i.e. with the opposite phase as the modulation predicted by the D01 model.

\section{Observations}

In the course of our campaign, we have observed $\beta$ Cep with both Chandra and XMM-Newton. In both cases, the proposed observational strategy was to perform 4 observations, as close as possible to the phases $0.0,0.25,0.50$, and 0.75 . Phases 0.0 and 0.50 correspond to the magnetic equator being observed face-on, while phases 0.25 and 0.75 correspond to the edgeon configuration. In the MCWS scenario, these 4 observations should ensure maximum modulation of the X-ray emission.

The XMM-Newton observatory allows simultaneous operation of the RGS high-resolution spectrograph and the EPIC cameras. The goal of the XMM-Newton observation was to detect modulation in the spectral parameters, namely the global spectral temperature and emission measure as observed in the EPIC low-resolution CCD spectra, and in the triplet ratios from the RGS spectra. The triplet ratios, as discussed below, are a diagnostic, for early-type stars, of the distance at which the emitting plasma is located from the star.

The Chandra observation was performed using the LETGS spectrograph, with the aim of detecting whether the lines would be broadened asymmetrically (as e.g. expected in a unconfined, self-shocked wind), or symmetrically (as expected if significant rotational broadening is present), or whether modulation of the radial velocity with the star's rotational period is present (as expected e.g. in the presence of strongly localized plasma).

In practice, a number of constraints on the scheduling of space observatories made it difficult to schedule the observations precisely at the phases desired. The Chandra observations were particularly critical because of issues with the spacecraft thermal control, so that in practice the phase coverage is, especially for the Chandra data, not optimal - see Table 2 for the phase coverage of the XMM-Newton observations, and Table 6 for the Chandra observations.

\subsection{XMM-Newton data reduction}

Each of the 4 XMM-Newton observations was processed individually with the standard SAS V.6.0 pipeline. We reduced and analyzed the RGS (1 and 2) data, as well as the EPIC MOS and pn data. We report only the results obtained for the pn data, since these were consistent with the MOS data (with one exception noted in the text). Some periods of high background (proton flaring) were present in the observations, so that the EPIC data were screened to exclude them. We used as a criterion the number 
of events detected at energies over $10 \mathrm{keV}$ in the entire frame, and we discarded data periods in which this rate was higher than 12 per minute. The RGS1 and RGS2 spectra were analyzed separately, using the "PintOfAle" package (Kashyap \& Drake 2002) to determine the line fluxes.

Given the accuracy of the pointing, we found that it was possible to use the same source and background regions for all four observations.

\subsection{Chandra data reduction}

The Chandra data were reduced using the standard pipeline procedures in the latest release of the data reduction package, CIAO (v.3.3.0.1). Barycentric corrections were applied to the timing in the reduced files, accounting and correcting for the spacecraft motion. Background-subtracted spectra and light curves were extracted from four separate event files, one for each Chandra observation. X-ray light curves were created by defining source and background regions and dividing the background region count rate by the relevant scaling factor prior to background subtraction. The pointing stability of Chandra was also sufficient to enable the use of the same background and source regions for all four exposures.

Four sets of spectra were extracted, one for each of the four observations, integrating over the full exposure in each observation. Since we want to measure centroid positions accurately and individual lines are detected with limited $S / N$, we co-add the spectra from observations taken at similar phases (the first two data sets were centered close to phase $\sim 0$ and the other 2 close to phase 0.25$)$. Since the LETG wavelength scale is affected by non-linear deviations from the laboratory positions of the wavelengths (Chung et al. 2004), the +1 and -1 orders may show systematic differences. To ensure that we can distinguish between instrumental variability and genuine variability in the target system, we consider the +1 and -1 orders separately in the subsequent analysis. We use the +1 and -1 order profiles to test for consistency and evaluate the precision of any shifts in centroid position measurements from one observation to another.

\section{Results}

\subsection{EPIC data}

We first inspected the background-subtracted light curves from the pn detector, shown in Fig. 3. Small amplitude variability is probably present in all 4 observations, since a formal Kolmogorov-Smirnov (KS) test indicates a high probability that the source has undergone some variability during the 4th segment (bottom right panel of Fig. 3). On the other hand, during the 3rd segment of the observation (bottom left panel of Fig. 3), the KS test gives a very low probability of the source being variable, consistent with a lack of actual variations during the observation.

We also searched for evidence of modulation in the intensity of the X-ray emission at the $4 \mathrm{~h} 34 \mathrm{~m}$ pulsation period of $\beta$ Cep, as reported by Cohen (2000). A period search performed on the complete pn data set using a $\chi^{2}$ test on the phase-folded data set failed to detect any significant periodicity. In particular, no periodicity was evident at the $4 \mathrm{~h} 34 \mathrm{~m}$ pulsation period. Inspection of the $4 \mathrm{pn}$ individual light curves phase-folded at the pulsation period does not show any evidence of modulation in any of them. Furthermore, any possible hint of a modulation is at a different phase in each of the 4 light curves. This, and the fact that Cohen (2000) dit not performed a period search, but rather assumed the period and fitted the amplitude and phase, makes it likely that the period reported by Cohen (2000) is spurious.

We have fitted the 4 individual EPIC pn spectra with variable abundance thermal models. To obtain a satisfactory fit, the spectra require two temperature components. The lower temperature component at $T \simeq 0.25 \mathrm{keV}$ is dominant, with an emission measure in all cases more than one order of magnitude larger than the hotter temperature one. The hotter temperature is not well constrained, due to the low statistics of the high-energy tail in the spectra, and it ranges between 0.7 and $2.0 \mathrm{keV}$. Abundances are also not strongly constrained, and appear to be, if anything, moderately depleted with respect to the photospheric abundances of $\beta$ Cep. The best fit values reported in Table 2 show some evidence for variations in the abundance among the four spectra, in particular for Si. While the formal robustness of such fluctuations is modest, the visual appearance of the pn spectra for the first and second observation (Fig. 4) is strikingly different: the Si complex at $\simeq 1.9 \mathrm{keV}$ is prominent in the first observation and almost absent in the second one.

The level of photometric variability among the 4 observations is modest (of order 10\%), which allows us to perform a joint spectral analysis of all four spectra simultaneously. The results are reported in Table 3. Given the availability of photospheric abundances for $\beta$ Cep (Morel et al. 2006, see Table 1), we can determine the ratio of coronal to photospheric metallicity for $\beta$ Cep itself, without reference to the solar metallicity, as reported in Table 1 . Only 3 elements $(\mathrm{O}, \mathrm{Si}, \mathrm{Fe})$ have both coronal and photospheric abundances available, and again, moderate metal depletion in the X-ray emitting plasma with respect to the stellar photosphere is present.

The $N(\mathrm{H})$ resulting from the fit $\left(2.5 \times 10^{20} \mathrm{~cm}^{-2}\right)$ corresponds (using the relation $N(\mathrm{H}) / A_{\mathrm{V}}=1.9 \times 10^{21}$ ) to an interstellar absorption of $0.13 \mathrm{mag}$. This is consistent with the published values for the interstellar reddening toward $\beta \operatorname{Cep}\left(E_{B-V} \leq 0.04\right.$, corresponding to $\left.A_{\mathrm{V}} \leq 0.13\right)$.

In the ROSAT PSPC band $(0.1-2.0 \mathrm{keV})$, the best-fit model for the pn data has a flux corresponding to an X-ray luminosity of $4.0 \times 10^{30} \mathrm{erg} \mathrm{s}^{-1}$, and shows little long-term variability compared with the 1990 ROSAT observation, which had an X-ray luminosity of $4.2 \times 10^{30} \mathrm{erg} \mathrm{s}^{-1}$.

\subsection{RGS data}

We first analyzed the 4 individual RGS spectra separately. Given the moderate $S / N$ of the spectra, no difference is visible among the different spectra, as shown in Fig. 7 for the region of the $\mathrm{O}$ VII triplet. We therefore co-added all the spectra and limited the further analysis to this co-added spectrum. The coadded spectrum is shown in Fig. 8, again limited to the O VII triplet region.

The RGS spectral range includes a number of He-like triplets, which provide useful diagnostics of the plasma conditions. The most relevant at the plasma temperature of $\beta$ Cep are those from N VI (at $29 \AA$ ), O VII (at $22 \AA$ ), and Ne IX (at $13.5 \AA$ ), each composed of 3 spectral features: the resonance line $r$, the intercombination doublet $i$ (which is blended into a single observed line), and the forbidden line $f$. The intensity ratio $f / i$ is determined by both the plasma density (above a certain critical density which changes from element to element) and the ambient UV field. The $f$ line is the result of a decay from a metastable state; at low density and in the absence of an ambient radiation field, the metastable state will decay undisturbed and the $f$ line will be at maximum strength. Both collisions and radiative 

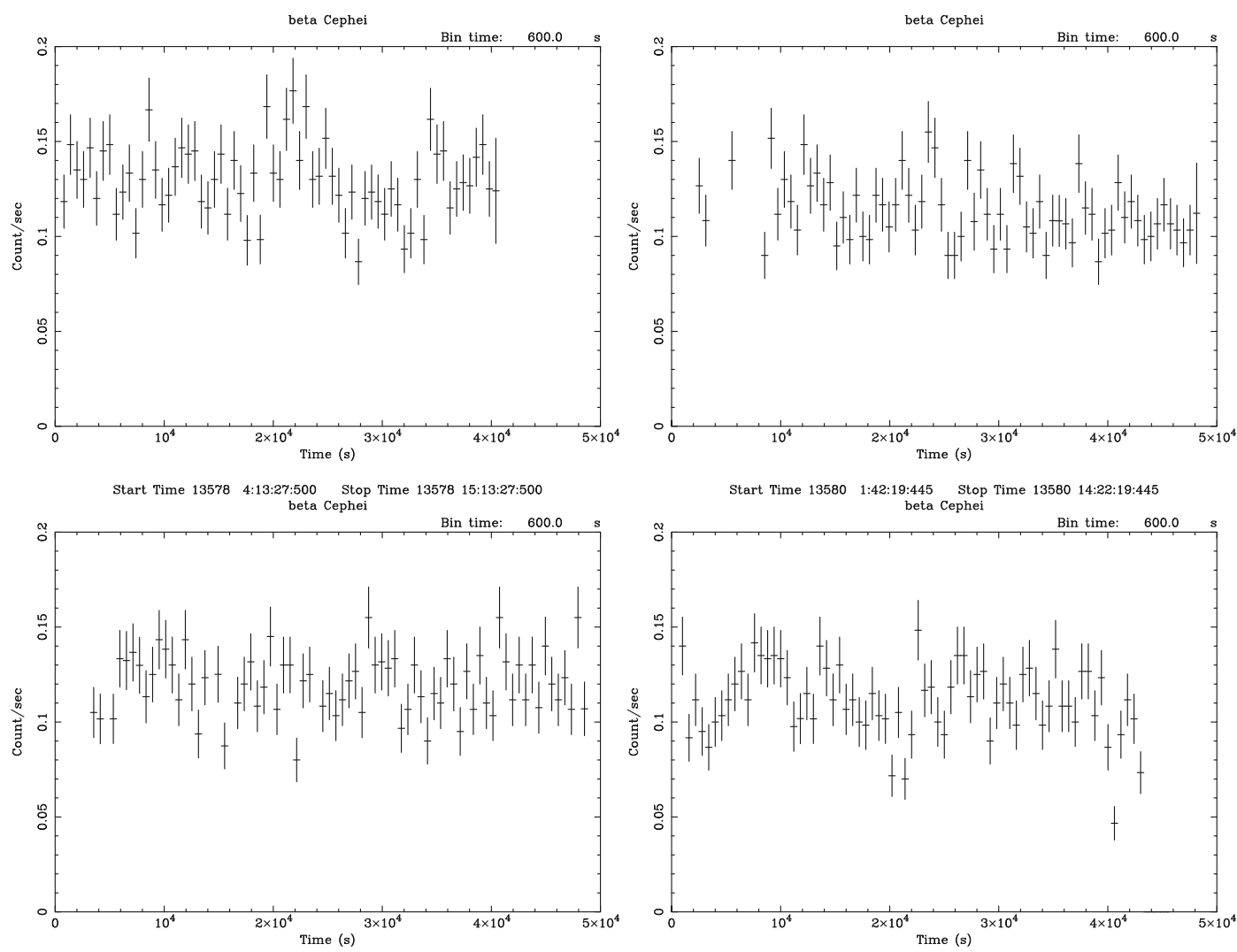

Start Time 13584 5:58:56:924 Stop Time 13584 18:28:56:924

Start Time 13588 5:16:43:106 Stop Time 13588 17:36:43:106

Fig. 3. The 4 light curves from the EPIC (pn) observations, background subtracted and binned at $1200 \mathrm{~s}$ resolution.
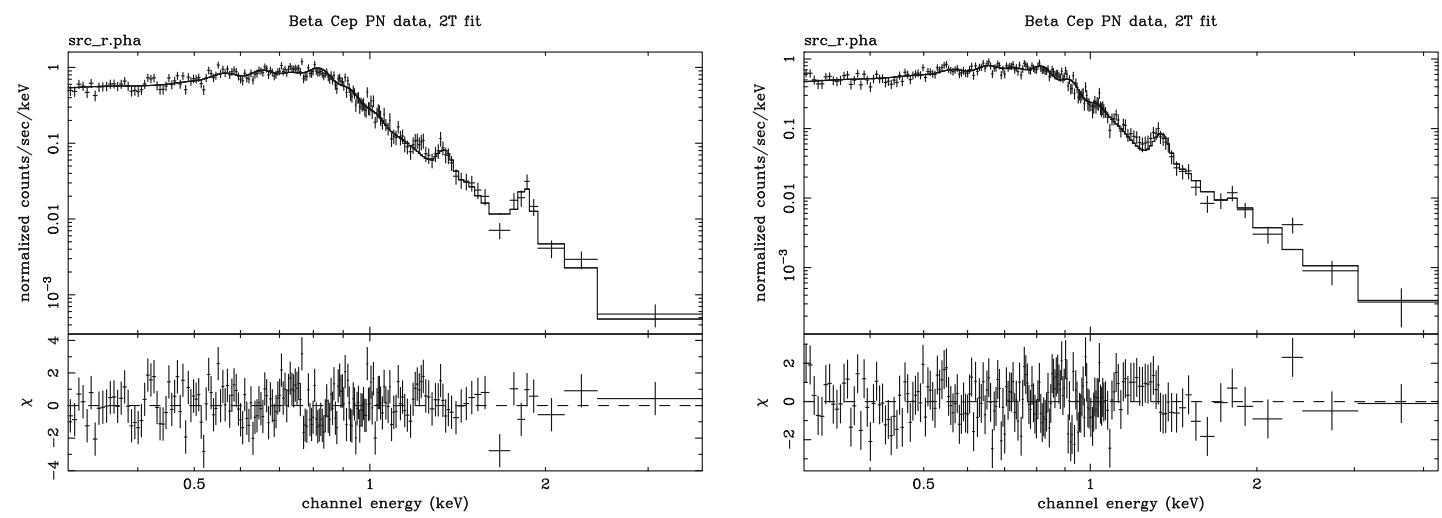

Fig. 4. Two-temperature, variable abundance fits to the EPIC pn spectra obtained in the first (left panel) and second (right panel) observations of $\beta$ Cep. The relevant best fit parameters are presented in Table 2.

pumping can depopulate the metastable state, so that an increase in either the density or ambient radiation field will result in a less intense $f$ line. For the triplets of interest, the radiative pumping depopulating the metastable state is due to ambient UV photons. While in cool stars the $f / i$ ratio is commonly used as a diagnostic of plasma density, in hot stars (such as $\beta$ Cep) the ambient UV field due to the star's photospheric emission is strong and dominates the observed $f / i$ ratio for the typical plasma densities. The $f / i$ ratio depends on both density and the UV field as described by the relation

$$
R=R_{0} \frac{1}{1+\phi / \phi_{\mathrm{c}}+n_{\mathrm{e}} / n_{\mathrm{c}}}
$$

where $R_{0}$ is the $f / i$ ratio in the limit of low-density and negligible UV field, $n_{\mathrm{e}}$ is the electron density of the plasma, $n_{\mathrm{c}}$ is the critical density (approximately, for N VI, $5 \times 10^{9}$, for O VII $3 \times 10^{10}$ and for Ne IX $\left.6 \times 10^{11} \mathrm{~cm}^{-3}\right), \phi$ is the UV photoexcitation rate of the relevant transition (which depends on the ambient UV radiation field due to the star's photospheric emission, at the wavelength of 1906, 1637, and $1270 \AA$ for N VI, O VII, and Ne IX respectively), and $\phi_{\mathrm{c}}$ is the rate at which $R=R_{0} / 2$. To determine the photoexcitation rate $\phi$, we accounted for the variation of the UV flux with distance from the photosphere, using the formalism of Mewe \& Schrijver (1978) who include this effect in the so-called dilution factor.

Under the assumption that the plasma is below the critical density $n_{\mathrm{c}}$, the observed $f / i$ ratio depends only on the distance 
Table 3. The joint best-fit spectral parameter for the $2-T$ fit with individually varying metal abundances for the $4 \mathrm{pn}$ spectra. The notation " $M / M_{\odot}$ " indicates the ratio between the $\beta$ Cep coronal abundance and the solar photospheric abundance. The notation " $M / M_{\text {phot }}$ " indicates the ratio between the coronal abundance of $\beta$ Cep determined here and the photospheric abundance of $\beta$ Cep determined by Morel et al. (2006). In all cases, the solar photospheric abundances of Grevesse \& Sauval (1998) were used. The indicated uncertainties are $90 \%$ errors derived from a $\Delta \chi^{2}$ analysis. For all heavy elements not explicitly indicated, the abundances are equal to the value for $\mathrm{Fe}$.

\begin{tabular}{cl}
\hline \hline$N(\mathrm{H})$ & $2.50 \pm 0.01 \times 10^{20} \mathrm{~cm}^{-2}$ \\
$T_{1}$ & $0.24 \pm 0.01 \mathrm{keV}$ \\
$T_{2}$ & $0.63 \pm 0.03 \mathrm{keV}$ \\
$E M_{1}$ & $1.1 \pm 0.210^{54} \mathrm{~cm}^{-3}$ \\
$E M_{2}$ & $1.3 \pm 0.310^{53} \mathrm{~cm}^{-3}$ \\
$\mathrm{O} / \mathrm{O}_{\odot}$ & $0.12 \pm 0.02$ \\
$\mathrm{Ne} / \mathrm{Ne}_{\odot}$ & $0.18 \pm 0.03$ \\
$\mathrm{Si} / \mathrm{Si}_{\odot}$ & $0.31 \pm 0.06$ \\
$\mathrm{Fe} / \mathrm{Fe}_{\odot}$ & $0.33 \pm 0.04$ \\
$\mathrm{O} / \mathrm{O}_{\text {phot }}$ & 0.28 \\
$\mathrm{Si} / \mathrm{Si}_{\text {phot }}$ & 0.86 \\
$\mathrm{Fe} / \mathrm{Fe}_{\text {phot }}$ & 0.60 \\
\hline
\end{tabular}

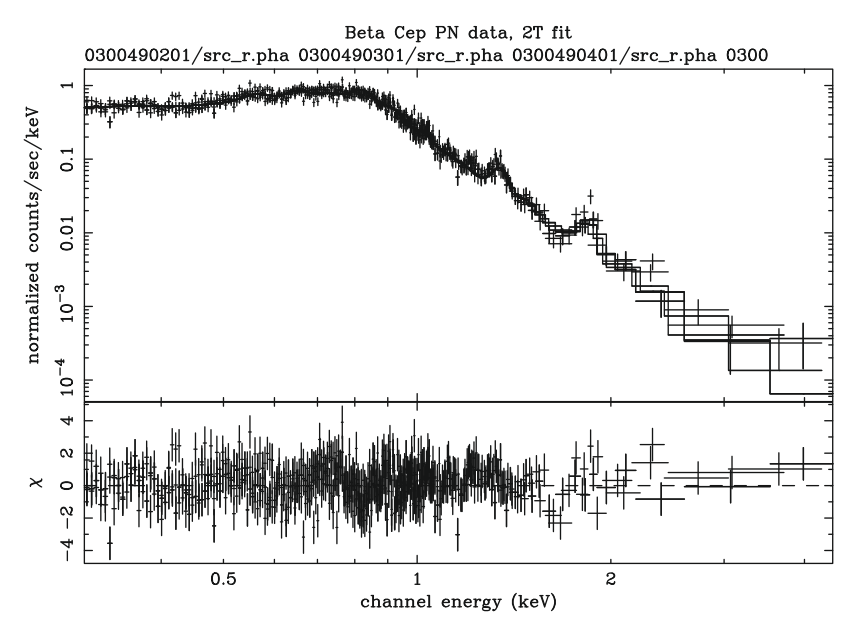

Fig. 5. Joint two-temperature, variable abundance fit to the 4 pn spectra of $\beta$ Cep.The relevant best fit parameters are presented in Table 3.

from the photosphere and can therefore be used to indicate the characteristic distance at which the bulk of the emitting plasma is located. Given that the density predicted by the MCWS model is below $10^{9} \mathrm{~cm}^{-3}$ we expect this assumption to be verified.

We perform the analysis of the 3 triplets visible in our spectra, namely N VI, O VII and Ne IX. The fluxes of all major lines used in our analysis are reported in Table 4. For the $\mathrm{N}$ VI triplet, no forbidden line is detected in the spectrum. The upper limit to its flux translates into an upper limit on the $f / i$ ratio and therefore the characteristic distance of the plasma from the photosphere.

The Ne IX triplet is affected significantly by blending, and the blending lines are unresolved at XMM-Newton resolution. While the forbidden line is relatively isolated, the intercombination line is strongly affected by blending from Fe lines, which would require high $S / N$ and resolution to be resolved completely (e.g. Ness et al. 2003). In our spectra, the $f$ line is undetected, which provides an upper limit to the characteristic distance from the star.

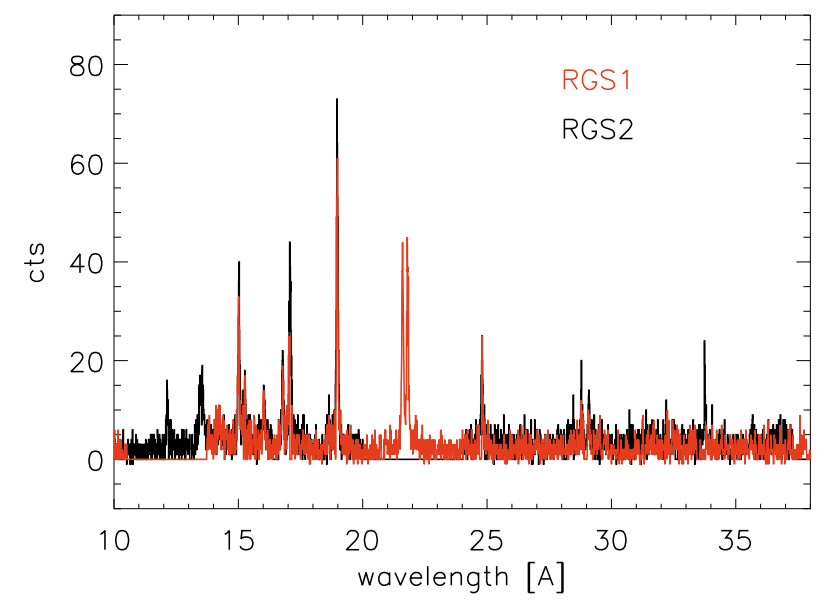

Fig. 6. The RGS1 (red) and RGS2 (black) spectra. All 4 individual spectra have been coadded in this plot.

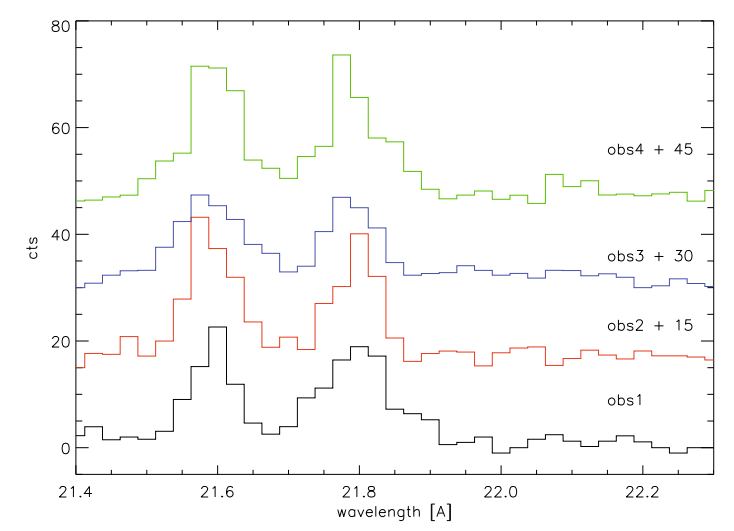

Fig. 7. The 4 individual RGS1+RGS2 spectra in the region of the $\mathrm{O}$ VII triplet. The spectra have been offset in the vertical direction for clarity.

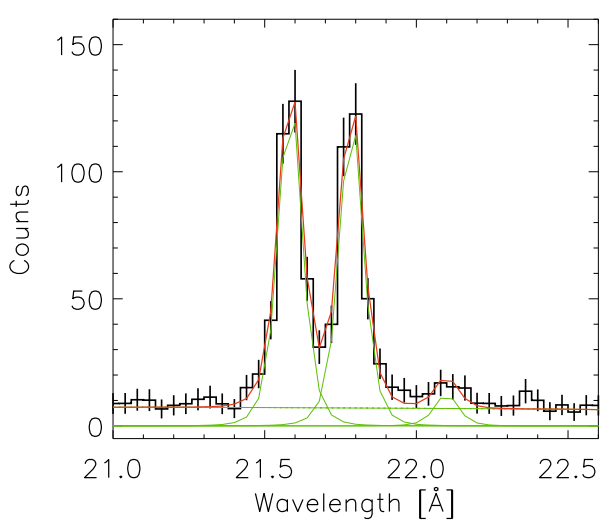

Fig. 8. The 4 coadded RGS1+RGS2 spectra in the region of the O VII triplet, with the fit to the 3 lines plus a constant.

In the OVII triplet, the forbidden line is detected, at the $\simeq 3 \sigma$ level, in the coadded spectra (Fig. 8), which corresponds to a measurement of the ratio $f / i=0.095 \pm 0.033$.

To determine the influence of the photospheric UV flux on the X-ray triplets, a measure of the star's UV field is required. The simplest approach is to use the star's effective temperature (26000 K) and, under the assumption of a black body emitter, compute the corresponding UV flux at the wavelength of interest. A more empirical approach relies on the measurement of the 
Table 4. The line fluxes measured in the RGS spectrum. Fluxes are in $10^{-6}$ photons $\mathrm{cm}^{-2} \mathrm{~s}^{-1}$.

\begin{tabular}{rrrrr}
\hline \hline Det. & Line ID & $\lambda$ & Flux & Error \\
\hline RGS2 & Ne X Ly $\alpha$ & 12.132 & 10.6 & 1.7 \\
RGS2 & Ne IX $r$ & 13.447 & 10.4 & 1.7 \\
RGS2 & Ne IX $i$ & 13.550 & 8.8 & 1.7 \\
RGS2 & Ne IX $f$ & 13.700 & 1.6 & 0.9 \\
RGS2 & Fe XVII & 15.010 & 21.7 & 2.2 \\
RGS2 & Fe XVII & 15.190 & 6.1 & 1.4 \\
RGS2 & Fe XVII & 15.260 & 8.9 & 1.6 \\
RGS2 & Fe XVIII+O VIII Ly $\beta$ & 16.010 & 6.6 & 1.9 \\
RGS2 & Fe XVII & 16.070 & 3.6 & 1.9 \\
RGS2 & Fe XVII & 16.780 & 17.7 & 2.0 \\
RGS2 & Fe XVII & 17.050 & 20.8 & 3.1 \\
RGS2 & Fe XVII & 17.096 & 18.9 & 3.0 \\
RGS2 & O VII & 18.627 & 6.8 & 1.5 \\
RGS2 & O VIII Ly $\alpha$ & 18.970 & 59.4 & 3.2 \\
RGS1 & O VII $r$ & 21.600 & 45.1 & 3.4 \\
RGS1 & O VII $i$ & 21.800 & 41.3 & 3.2 \\
RGS1 & O VII $f$ & 22.100 & 4.8 & 1.9 \\
RGS2 & N VII Ly $\alpha$ & 24.780 & 21.2 & 2.2 \\
RGS2 & N VI $r$ & 28.790 & 18.4 & 2.3 \\
RGS2 & N VI $i$ & 29.080 & 17.2 & 2.3 \\
RGS2 & N VI $f$ & 29.530 & 2.4 & 1.8 \\
\hline
\end{tabular}

Table 5. The unabsorbed UV flux at Earth and intensity at the stellar photosphere, at the wavelength relevant to the radiative pumping of the $f$ transition for the N VI, O VII, and Ne IX triplets, derived from an IUE spectrum of $\beta$ Cep. $F_{\lambda}$ in units of $\mathrm{erg} \mathrm{cm}^{-2} \mathrm{~s}^{-1} \AA^{-1}, I_{\lambda}$ in units of $\operatorname{erg~cm}{ }^{-2} \mathrm{~s}^{-1} \AA^{-1} \mathrm{sr}^{-1}$.

\begin{tabular}{lcccc}
\hline \hline & $E_{B-V}$ & N VI $(1906 \AA)$ & O VII $(1637 \AA)$ & Ne IX $(1270 \AA)$ \\
\hline$F_{\lambda}$ & 0.00 & $3.8 \times 10^{-9}$ & $5.6 \times 10^{-9}$ & $1.1 \times 10^{-8}$ \\
$I_{\lambda}$ & 0.00 & $1.1 \times 10^{9}$ & $1.6 \times 10^{9}$ & $3.2 \times 10^{9}$ \\
$\mathrm{~T}$ & 0.00 & 19995 & 21227 & 23969 \\
$F_{\lambda}$ & 0.04 & $5.1 \times 10^{-9}$ & $7.4 \times 10^{-9}$ & $1.6 \times 10^{-8}$ \\
$I_{\lambda}$ & 0.04 & $1.5 \times 10^{9}$ & $2.1 \times 10^{9}$ & $4.6 \times 10^{9}$ \\
$\mathrm{~T}$ & 0.04 & 21674 & 22701 & 25899 \\
\hline
\end{tabular}

UV flux at the wavelengths of interest based on e.g. IUE spectra. We compared the results obtained using both approaches.

To determine the UV flux at the 3 wavelengths of interest, we retrieved from the public archive an IUE observation of $\beta$ Cep, from which the flux at Earth was determined. To derive the radiation intensity at the stellar surface, the flux needs correcting for the effects of interstellar extinction. Extinction toward $\beta$ Cep is modest, and the values in the literature range from $E_{B-V}=0.04$ (Kontizas \& Theodossiou 1980) to $E_{B-V}=0.01$ (Krelowski \& Sneden 1993). To assess the sensitivity of our analysis to the value of interstellar extinction, we considered the case of both $E_{B-V}=0.04$ and no measurable extinction. Using the UV interstellar extinction law reported by Zombeck (1990), we determined the extinction at each of the 3 wavelengths, determined the radiation intensity, and derived the equivalent blackbody temperature.

The dependence of the $f / i$ ratio on the distance from the star's photosphere is plotted in Fig. 9, for the three different assumptions regarding the photospheric UV flux (black body, IUE spectrum with $E_{B-V}=0.00$, IUE spectrum with $\left.E_{B-V}=0.04\right)$. For each triplet, the range of $f / i$ determined from the observed spectrum is indicated by a thicker line. For N VI and Ne IX, an upper limit to the location of the plasma can be determined, of respectively $\simeq 15-25 R_{\star}$ for $\mathrm{N}$ VI and $\simeq 2.5-3 R_{\star}$ for Ne IX,

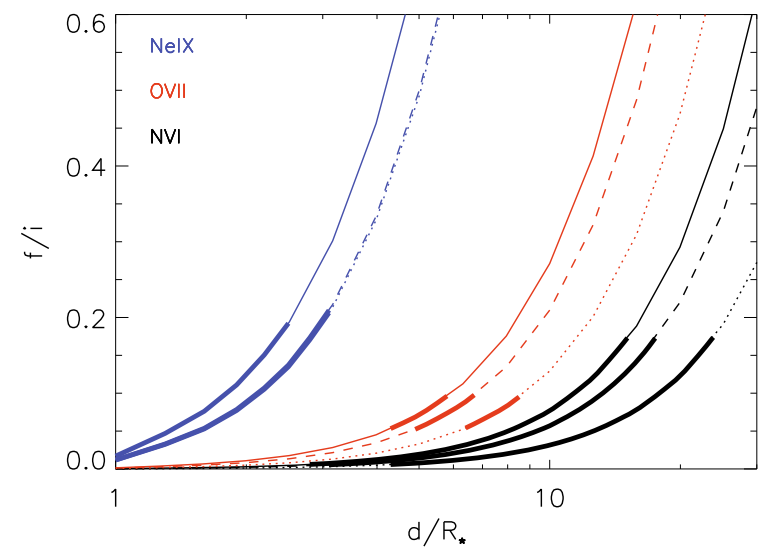

Fig. 9. The thick lines show the measured $1 \sigma$ range for the $f / i$ line ratios, and indicate the allowed distance for the location of the X-ray emitting plasma for the Ne IX, O VII and N VI triplets. The continuous curve corresponds to the UV flux determined from the IUE spectrum with $E_{B-V}=0.00$, the dashed curve to the same with $E_{B-V}=0.04$, and the dotted curve to the UV flux corresponding to a $26000 \mathrm{~K}$ blackbody.

depending on the assumption for the UV field. In the following, we adopt the curve derived using the UV flux determined from the IUE spectrum and $E_{B-V}=0.04$.

For $\mathrm{O}$ VII, the characteristic distance of the emitting plasma from the photosphere is between $\simeq 3$ and $\simeq 5 R_{\star}$ using the UV flux determined from the IUE spectrum and $E_{B-V}=0.04$. If the $\mathrm{O}$ VII emitting plasma is located at an effective distance from the photosphere of $\simeq 5 R_{\star}$, the rotational velocity of the plasma, assuming rigid rotation at the star's rotational period of $12 \mathrm{~d}$, would be $190 \mathrm{~km} \mathrm{~s}^{-1}$, decreasing to $130 \mathrm{~km} \mathrm{~s}^{-1}$ for an effective distance of $\simeq 4 R_{\star}$. This velocity is below the velocity resolution of the Chandra LETG/HRC instrument and therefore we expect (as indeed observed) that the spectral lines in the LETG spectrum are consistent with the instrumental profile.

The RGS spectrum also allows us to easily derive abundance ratios by taking the ratios of lines of different elements characterized by similar emissivities as a function of temperature (Drake $\&$ Testa 2005). Using the Ne X Ly $\alpha$ at $12.132 \AA$, Ne IX $r$ line at $13.447 \AA$, and the $\mathrm{O}$ VIII Ly $\alpha$ line at $18.970 \AA$ we derived a ratio $\mathrm{Ne} / \mathrm{O}$ of $0.32 \pm 0.05$ (about twice the solar ratio of $0.18 \mathrm{e} . \mathrm{g}$. from Grevesse \& Sauval 1998), marginally consistent within the error bars with the ratio derived from the pn spectrum $(\simeq 0.67)$.

\subsection{Chandra/LETG observations}

The main aim of the Chandra LETG observation of $\beta$ Cep was to study the X-ray line profiles at the highest possible spectral resolution for the low-temperature plasma of $\beta$ Cep, with the aim of detecting either a modulation in the line centroid or a broadening in the line profile. In the MCWS scenario, modulation in the line centroid in phase with the rotation period would be expected if the plasma is distributed non-homogeneously around the star and at sufficient distance from the star so that the rotational velocity is visible as Doppler shift.

If on the other hand the plasma is distributed uniformly, no velocity shifts are expected but stellar rotation induces a broadened line profile. Assuming for $\beta$ Cep that $R=6.5 R_{\odot}$ and the rotational period is $12 \mathrm{~d}$, the velocity of a plasma orbiting around the star will be $v=28 \times r \mathrm{~km} \mathrm{~s}^{-1}$, where $r$ is the distance of the plasma from the stellar center expressed in stellar radii.

For this purpose, we obtained four Chandra observations of $\beta$ Cep (ObsIDs 5395, 6138, 7194, and 7195) using the low 
Table 6. The observation log of the Chandra data sets.

\begin{tabular}{lllllll}
\hline \hline ObsID & $\begin{array}{l}\text { Exposure } \\
\text { ks }\end{array}$ & $\begin{array}{l}T_{\text {start }} \\
\text { JD }\end{array}$ & $\begin{array}{l}T_{\text {end }} \\
\text { JD }\end{array}$ & $\phi_{\text {start }}$ & $\phi_{\text {end }}$ & $\begin{array}{l}\text { Count rate } \\
\text { cts/s }\end{array}$ \\
\hline 5395 & 36.43 & 2453674.4519389 & 2453674.8897456 & 0.0096 & 0.0461 & 0.086 \\
6138 & 29.54 & 2453675.0851294 & 2453675.4490259 & 0.0624 & 0.0927 & 0.089 \\
7194 & 38.17 & 2453677.1208892 & 2453677.5798238 & 0.2320 & 0.2702 & 0.082 \\
7195 & 27.15 & 2453677.7494105 & 2453678.0855105 & 0.2844 & 0.3124 & 0.075 \\
\hline
\end{tabular}

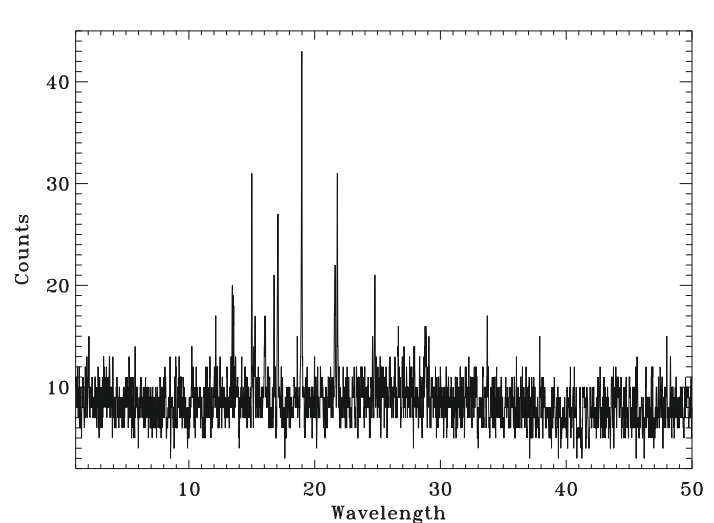

Fig. 10. The LETG spectrum of $\beta$ Cep obtained by summing the four Chandra observations discussed here.

energy transmission grating (LETG) with the high-resolution camera spectroscopic array (HRC-S). The phases spanned by the four Chandra exposures are listed in Table 6, computed using the ephemeris of D01. While the original plan was to obtain 4 observations at phases as close as possible to $0.0,0.25,0.5$, and 0.75 , restrictions on Chandra operations limited the accessible phases. As Table 6 shows, two datasets were taken close to phase 0.0 and the final two observations were acquired close to phase 0.25 , providing a more limited phase sampling than originally planned but still allowing the system to be studied in both face-on and edge-on configurations. The resulting LETG spectrum, obtained by summing all the four observations, is shown in Fig. 10.

The LETG/HRC-S configuration enables precise measurement of velocity shifts in X-ray spectra as the instrumental $F W H M$ is $0.057 \AA$, which corresponds to a velocity $F W H M$ of approximately $900 \mathrm{~km} \mathrm{~s}^{-1}$ beyond a wavelength of $19 \AA$. As shown e.g. by Hoogerwerf et al. (2004), the precision with which the centroid of a line profile can be measured depends strongly on the peak number of counts in the line profile. By adding the counts obtained over two exposures centered about similar phases, we can maximise our centroiding ability. Given the count rate of $\beta$ Cep and the exposure times, our measurement error in the centroid position of the strongest unblended line profile, O VIII $18.97 \AA$, for observations 1 and 2 (centered at phases 0.05 and 0.27 respectively) corresponds to $\sim 160 \mathrm{~km} \mathrm{~s}^{-1}$.

The spatial resolution of Chandra can easily resolve $\beta$ Cep from its companion at 13.4 arcsec. Visual inspection of the Chandra image indicates that no X-ray emission is visible from the companion and that the X-ray source is coincident with the optical position of $\beta$ Cep to within a fraction of arcsec. The speckle companion at 0.1 arcsec is on the other hand unresolved.

We first extracted the light curve of the Chandra observation, searching for evidence of rotational modulation in the X-ray emission. The 4 light curves for the 4 observations are plotted in Fig. 11. The emission is constant across the 4 segments, showing, in a similar way to the XMM-Newton observation, no evidence of rotationally modulated X-ray emission. No significant flaring is present, and no systematic changes in the X-ray flux are observed between the different observation phases.

We then searched for evidence of rotational modulation in the radial velocity of the emitting plasma by analyzing both the centroid and profile of the $19 \AA$ O VIII line. Given the limited phase sampling afforded by the Chandra observations, we again summed the two observations close to phase 0.0 and the two observations close to phase 0.25 . No evidence of varying Doppler shift with phase is present in our observations: as shown in Table 7, the position of the line centroid is constant, and its nominal position does not vary within the error estimate. The same applies to the $F W H M$ of the line.

As mentioned above, the strongest unblended line profile in the dataset is the O VIII resonance doublet close to $18.97 \AA$. Gaussian fitting procedures in the data analysis package Sherpa were used to measure the centroid positions of the +1 and -1 order profiles. We found that any velocity shift in the line centroid position is compatible with the measurement errors (see Table 7). We conclude, therefore, that there is no measurable change in the radial velocity of the X-ray emitting plasma. Given the measurement errors of $0.01 \AA$, we place an upper limit on any relative velocity shifts of $\leq 160 \mathrm{~km} \mathrm{~s}^{-1}$ when comparing spectra centered at phases 0.05 and 0.27 . Furthermore, the O VIII $18.97 \AA$ line width is consistent with the instrumental broadening, which has a $F W H M=900 \mathrm{~km} \mathrm{~s}^{-1}$ (Table 7).

To investigate the level of broadening in the emission line profiles, we summed up the counts from the strongest line profiles in the spectral dataset. The strongest six line profiles all showed peak intensities of more than 15 counts; these were used to construct a composite profile to investigate the origin of the $\mathrm{X}$-ray emission from the system. This profile was built by first measuring the zero-velocity positions of the line profiles using the entire spectrum computed by summing over all four exposures. This was valid since the source position remained within 1 or 2 pixels between all observations; the wavelength scale should therefore be consistent between all four pointings. After the zero-velocity positions were established, both the +1 and -1 order spectra were converted to velocity-space using these zerovelocity offsets; they were then interpolated to the same wavelength resolution $\left(187 \mathrm{~km} \mathrm{~s}^{-1}\right.$ or $0.0125 \AA$ ), which corresponded to a line at the mean wavelength $(20 \AA)$, and coadded. The wavelengths of the lines used are centered at: Fe XVII 15.014 $\AA$, Fe XVII 17.0510 ̊, O VIII 18.9725 Fe XVII, O VII $21.6015 \AA$, O VII $21.8036 \AA$, and N VI $24.7792 \AA$, respectively.

Figure 12 shows the composite profiles for orders \pm 1 compared with the combined instrumental profile of the six strongest lines (dashed line). This was produced by scaling the instrumental profile until it fitted the amplitude of the corresponding strong line of each of the six strong line profiles, interpolating the scaled instrumental profile to the same velocity scale used for the composite profile, and coadding all of these scaled, interpolated instrumental profiles to produce a composite instrumental 

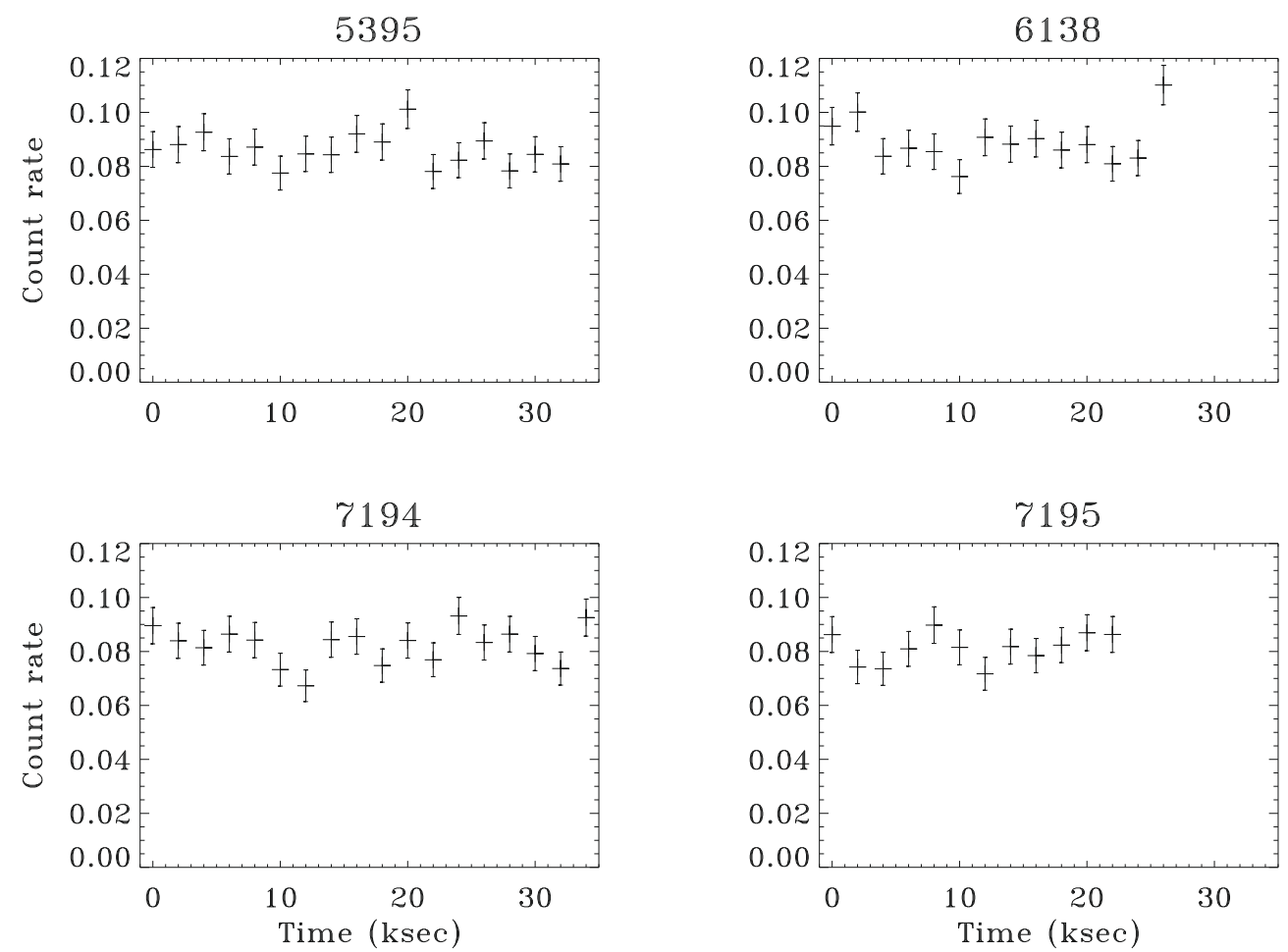

Fig. 11. The light curve of the 4 Chandra observations, integrated at $2 \mathrm{ks}$ binning. No evidence of modulation with the rotational period is present.

Table 7. The mean wavelength and $F W H M$ of the O VIII line, grouping observations into two groups of two and maximizing the phase sampling.

\begin{tabular}{lll}
\hline \hline Mean $\phi$ & $\lambda_{\mathrm{o}}(-1)[\AA]$ & $\lambda_{\mathrm{o}}(+1)[\AA]$ \\
\hline 0.05 & $18.976 \pm 0.010$ & $18.970 \pm 0.010$ \\
0.27 & $18.971 \pm 0.009$ & $18.977 \pm 0.006$ \\
\hline & $F W H M(-1)[\AA]$ & $F W H M(+1)[\AA]$ \\
\hline 0.05 & $0.07 \pm 0.03$ & $0.06 \pm 0.03$ \\
0.27 & $0.05 \pm 0.01$ & $0.05 \pm 0.02$ \\
\hline
\end{tabular}

profile. A similar analysis performed on phase-grouped spectra also failed to reveal any change in the line profile and positions.

We then analyzed the individual line profiles for evidence of velocity broadening, considering both the line profiles from the individual observations (Fig. 12) and the line profile obtained by summing all strong lines (Fe XVII 15.014 $\AA$, Fe XVII 17.0510 ̊, O VIII $18.9725 \AA$, O VII $21.6015 \AA$, O VII $21.8036 \AA$, and N VI $24.7792 \AA$ ) from all 4 observations. By assuming no variability among the observations this allows to obtain a much higher $S / N$ and thus to determine the line profile to a higher accuracy. The width of all the observed lines are consistent with the instrumental resolution $(\Delta \lambda \simeq 0.05 \AA$ for the LETG/HRC instrumental combination, and therefore allow the presence of plasma moving at velocities $\gtrsim 600 \mathrm{~km} \mathrm{~s}^{-1}$ to be excluded. In the case of rotational broadening this translates into a maximum distance from the stellar center of $d \lesssim 20 R_{\star}$. While not very restrictive, this provides an upper limit to the distance of the X-ray emitting plasma, which, in conjunction with the lower limits provided by the He-like triplet analysis, allows us to determine the location of the X-ray emitting region around the star.

The terminal wind velocity of $\beta$ Cep was estimated to be between $800 \mathrm{~km} \mathrm{~s}^{-1}$ (D01) and $1500 \mathrm{~km} \mathrm{~s}^{-1}$ (Schnerr et al. 2007); the lack of observed broadening points toward X-ray emission from a magnetically confined plasma, instead of a freely flowing wind.

We also verified that the $f / i$ ratios of the LETG spectra are consistent with the values derived for the RGS spectra. However, given the lower $S / N$ of the LETG data, the $f / i$ ratios have significantly higher associated uncertainties and therefore do not help in determining the location of the plasma.

\section{Discussion and conclusions}

\subsection{X-ray modulation and location of the plasma}

$\beta$ Cep has a close B6-8 companion, which could be the component on which the $\mathrm{H} \alpha$ emission is located (Schnerr et al. 2006). The companion is unlikely to contribute to the X-ray emission of the $\beta$ Cep system: in the regime in which $\mathrm{X}$-ray emission originates in shocks in the wind (i.e. for OB stars) the emission increases with stellar mass and spectral type; therefore the B1 primary is likely to dominate the X-ray emission from the system and thus all our conclusions are unaffected by the presence of the late $\mathrm{B}$-type companion and the fact that the $\beta$ Cep primary is not a Be star.

Our X-ray campaign on $\beta$ Cep provides limited evidence of modulation in its X-ray emission level, both short-term (i.e. within a rotational period) and long-term (i.e. from one observation to the other) with, for example, the ROSAT and XMM-Newton observations showing similar flux levels across several years. Evidence of low-level modulation of the X-ray emission with stellar rotation is present in the pn low-resolution X-ray data, while the LETG and RGS high-resolution spectra appear remarkably similar to each other. For instance, as shown in Fig. 7, the O VII triplet shows no evidence of variation from one rotational phase to the other. For the Chandra LETG spectra, little if any variability is present in the line fluxes and in the centroid of the line positions. 

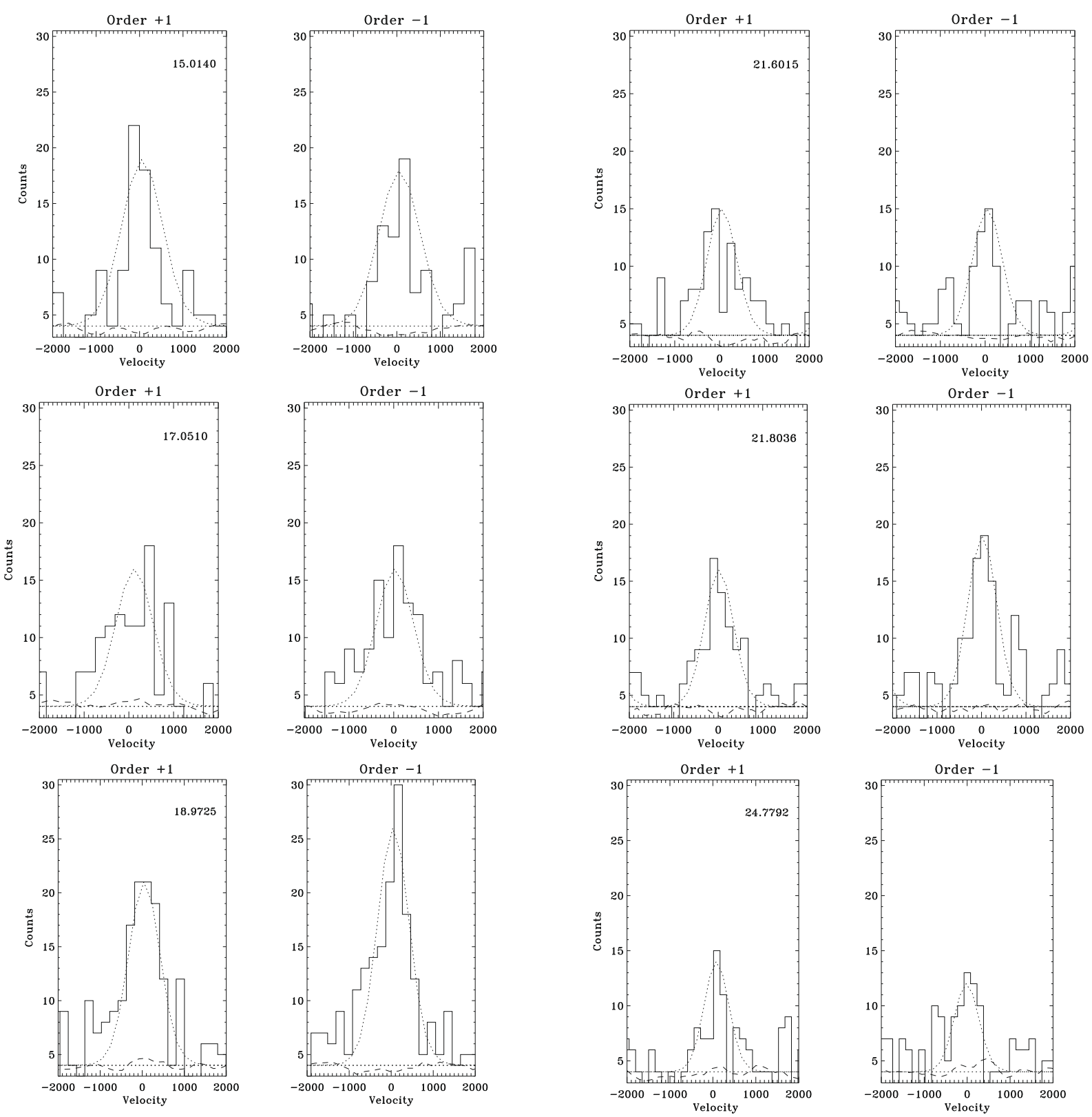

Fig. 12. The line profiles for the FeXVII 15.014 $\AA$, Fe XVII 17.0510 $\AA$, O VIII 18.9725 Fe XVII, O VII $21.6015 \AA$, O VII $21.8036 \AA$ and N VI 24.7792 Å lines respectively, obtained by adding all 4 Chandra observations.

Such a high degree of constancy in the X-ray emission contradicts the high $(\simeq 50 \%)$ level of rotational modulation expected in the MCWS framework of D01: with the geometry of $\beta$ Cep, the disk is observed alternatively face-one and edge-on. If X-ray emission is due to stationary shocks on either side of the disk (which is expected to have a high optical thickness at soft X-ray wavelengths), during face-on configurations about $50 \%$ of the flux should be absorbed.

In contrast to the expectations from the MCWS scenario by D01, we find a slightly $(\simeq 10 \%)$ higher emission level for the face-on configuration with respect to the edge-on configuration. This allows us to exclude the simple scenario proposed by D01 and in particular it excludes definitively the presence of the optically thick layer in the magnetic equator. Such a small modulation, with the observed phase, is consistent with the predictions of the dynamical MCWS models of ud-Doula \& Owocki (2002) and Gagné et al. (2005), which predict, for the $\beta$ Cep configuration, no thick disk and a modulation of approximately $5 \%$, fully consistent with the observed values.

The measurements of the line intensity in the He-like triplets, and in particular the O VII triplet, allows us to determine the location of the bulk of the emitting plasma. The formation temperature of the $\mathrm{O}$ VII triplet peaks at $0.17 \mathrm{keV}$, which does not differ significantly from the characteristic temperature of the bulk of the emitting plasma, as determined from the pn spectra. Therefore, the characteristic distance from the stellar photosphere determined using the O VII triplet should be representative of the majority of plasma at X-ray temperatures. The detection at $\sim 3 \sigma$ of the $f$ line in the O VII triplet allows us to determine a distance range from the photosphere (rather than an upper limit), estimated to be $d \simeq 4 R_{\star}$ (see Sect. 4.2). The derived location for the X-ray emitting plasma is also consistent with that predicted by both the ud-Doula \& Owocki (2002) and Gagné et al. (2005) models, which locate the plasma 

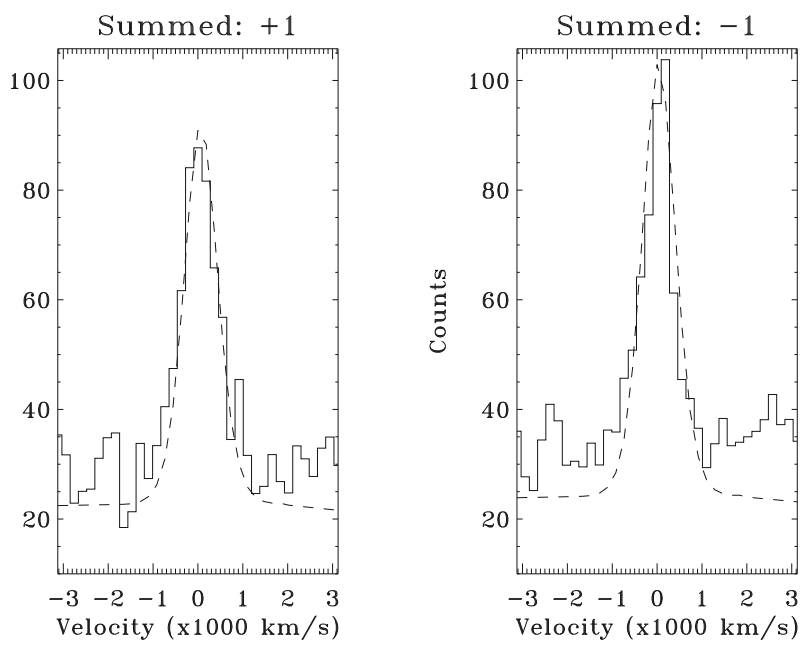

Fig. 13. The summed line profiles obtained adding up the Fe XVII $15.014 \AA$ A, Fe XVII $17.0510 \AA$ A, O VIII $18.9725 \AA$, O VII $21.6015 \AA$, O VII $21.8036 \AA$ and N VI $24.7792 \AA$ lines from the 4 Chandra observations. The dotted line shows the composite instrument profile.

between the Alfven and Kepler radii, i.e. between $R \simeq 5 R_{\star}$ and $R \simeq 7 R_{\star}$.

An additional constraint on the spatial location of the X-ray plasma in $\beta$ Cep is provided by the relation between the plasma pressure and the confining magnetic field: under the assumption that the X-ray emitting plasma is indeed magnetically confined, which is in agreement with the absence of line broadening that is expected for a standard wind shock model. The plasma density can be derived from the emission measure, which can be calculated either from the global fit to the pn spectrum, or taking the emission in a given line. If we examine the flux in the strongest ( $r$ ) $\mathrm{O}$ VII line, we derive $E M=1.5 \times 10^{55} \mathrm{~cm}^{-3}$. Assuming that the plasma is confined within a spherical shell between 4 and $6 R_{\star}$ (as inferred from the triplet ratios), the emitting volume is $V=7.5 \times 10^{37} \mathrm{~cm}^{3}$, which corresponds to an average plasma density of $n=4.5 \times 10^{8} \mathrm{~cm}^{-3}$. Such low density is consistent with there being no effect of the density on the He-like triplets of the elements considered here. The magnetic field needed to confine this plasma (taking into account the average temperature $T \simeq 0.3 \mathrm{keV}$ ) is $B=\sqrt{8 \pi 2 n k T} \simeq 3 \mathrm{G}$. By assuming that the stellar magnetic field is dipolar, taking the most reliable estimate of the polar magnetic field, $355 \mathrm{G}$, and scaling the dipolar field as $d^{3}$ ( $d$ being the distance from the star), the magnetic field at $d=4 R_{\star}$ from the photosphere is $355 / 5^{4} \simeq 5 \mathrm{G}$, similar to the pressure required to confine the emitting plasma. This indicates that the emitting plasma is weakly confined by the magnetic field, with $\beta \simeq 1$.

Using the location of the plasma found above $\left(R_{\text {out }}=6 R_{\star}\right.$ and $R_{\text {in }}=4 R_{\star}$ ) and assuming a geometrically thin disk, we can estimate the variation in the X-ray flux between an edge-on and a face-on configuration to be approximately $7 \%$. This number is compatible within the error bars with the observed variation level. This scenario would exclude the presence of X-ray emitting plasma at high latitudes above the magnetic equator and is compatible with predictions of dynamical MCWS models.

\subsection{Magnetic confinement}

Standard models of X-ray emission from massive stars, which originates in shocks in an unconfined wind, predict both a general blue-shifting of the line and its broadening. The magnitude of the expected broadening is comparable to the wind terminal velocity, which in the case of $\beta$ Cep is $\simeq 800-1500 \mathrm{~km} \mathrm{~s}^{-1}$. The blue-shift depends on the amount of absorption of the red wing of the line, and is expected to be lower than the broadening.

The analysis of the LETG line profiles excludes any broadening larger than the instrumental line profile, which has a $F W H M$ of approximately $600 \mathrm{~km} \mathrm{~s}^{-1}$. It also excludes blue-shifts higher than $160 \mathrm{~km} \mathrm{~s}^{-1}$. While they represent weak constraints relative to the wind terminal velocity of $\beta \mathrm{Cep}$, they indicate a lack of significant bulk motions in the X-ray emitting plasma, and imply that the latter is being magnetically confined, excluding, for $\beta$ Cep, X-ray emission from shocks in an unconfined wind.

The relatively low temperature of the X-ray emitting plasma (with the dominant component being at $\approx 3 \mathrm{MK}$ ) and the absence of significant short-term variability (flares) point however to a lack of magnetic heating (i.e. due to magnetic reconnection): in active cool stars, the magnetic reconnection that dominates the heating of the plasma produces much higher temperatures and stochastic variability. The high temperatures observed for $\theta^{1}$ Ori $\mathrm{C}$ also points to the presence of magnetic field in some massive stars.

In the case of $\beta \mathrm{Cep}$, the magnetic heating has the apparent function of confining the plasma, which is likely to have been heated to the observed 3 MK by shocks. Also, the bulk of the $\mathrm{X}$-ray plasma appears to be confined by a relatively weak magnetic field, close to the limit at which the magnetic pressure becomes too weak to confine the plasma.

\subsection{Comparison with other stars}

The behavior of $\beta$ Cep observed in our Chandra and XMM-Newton observations appears to differ significantly from the other well studied example of a magnetically confined wind in a massive star, $\theta^{1}$ Ori $C$. In that case, the X-ray emission is strongly modulated at the rotational period, by approximately $50 \%$, as predicted by the MCWS model. At the same time, the observed plasma temperatures are, for $\theta^{1}$ Ori $\mathrm{C}$, far higher than for $\beta \mathrm{Cep}$, reaching $30 \mathrm{MK}$. Furthermore, the analysis of the triplets in $\theta^{1}$ Ori $\mathrm{C}$ indicates that the emitting plasma is located much closer to the star's photosphere than in the case of $\beta$ Cep. In $\theta^{1}$ Ori C, the bulk of the plasma is located at $\simeq 1.5 R_{\star}$; in contrast, in $\beta$ Cep the cooler plasma traced by the $\mathrm{O}$ triplet is located at $\simeq 5 R_{\star}$, while the hotter plasma traced by the Ne triplet is located at $\lesssim 2 R_{\star}$ from the photosphere, indicating a stratification of the plasma as a function of temperature. Finally, the metal abundances determined for the $\theta^{1}$ Ori $\mathrm{C}$ plasma are far higher than for $\beta$ Cep. In $\beta$ Cep, all elements appear to be depleted with the exception of $\mathrm{Si}$, while many elements are enhanced in the $\theta^{1}$ Ori $\mathrm{C}$ spectrum, implying that a different process is in operation.

Gagné et al. (2005) modeled the observed emission of $\theta^{1}$ Ori $\mathrm{C}$ using a dynamic version of the MCWS, and showed that both infall into and outflow out of the photosphere make it unlikely that the thick disk predicted by Babel \& Montmerle (1997a) would form. They interpreted the observed modulation, which goes in the opposite direction from that predicted by the D01 model (as also observed by us for $\beta$ Cep) as occultation of the X-ray emitting plasma (located, in their model, close to the equator) by the stellar photosphere.

A similar situation, in which the dynamics prevent the thick disk from forming, is possibly present in $\beta$ Cep: the X-ray emission would then be produced by a magnetically confined wind, 
for which the lower observed temperatures are explained by the lower wind velocity.

\subsection{Conclusions}

The Chandra and XMM-Newton observations that we have discussed have failed to display the signatures expected by the static MCWS model of D01, in particular the strong rotational modulation of the X-ray emission due to the presence of the cool disk around the star. However, the X-ray plasma appears to be confined, and a small amplitude modulation is visible in the XMM-Newton EPIC data. With the low temperature and the absence of flaring, this can be interpreted as emission from a magnetically confined wind but without the cool, optically thick disk predicted by the D01 model. The observed modulation is fully compatible with the emission scenario predicted by the dynamical MCWS models for a star that has similar characteristics to those of $\beta$ Cep.

Acknowledgements. The authors thank the XMM-Newton and Chandra observatories for their support in the scheduling of a complex phase locked observation. Also the support of L. Scelsi in the analysis of the XMM-Newton RGS data is gratefully acknowledged. The authors also thank the referee, M. Gagne, for a very detailed and useful report. This work has made use of the BeSS database, operated at GEPI, Observatoire de Meudon, France:

http: //basebe.obspm. fr.

\section{References}

Abt, H. A., Levato, H., \& Grosso, M. 2002, ApJ, 573, 359

Agrawal, P. C., Singh, K. P., Riegler, G. R., \& Stern, R. A. 1984, MNRAS, 208, 845

Asplund, M., Grevesse, N., \& Sauval, A. J. 2005, in Cosmic Abundances as Records of Stellar Evolution and Nucleosynthesis, ed. T. G. Barnes, III, \& F. N. Bash, ASP Conf. Ser., 336, 25

Babel, J., \& Montmerle, T. 1997a, ApJ, 485, L29

Babel, J., \& Montmerle, T. 1997b, A\&A, 323, 121

Berghoefer, T. W., Schmitt, J. H. M. M., \& Cassinelli, J. P. 1996, A\&AS, 118, 481

Bouret, J.-C., Donati, J.-F., Martins, F., et al. 2008, MNRAS, 389, 75

Cassinelli, J. P., Miller, N. A., Waldron, W. L., MacFarlane, J. J., \& Cohen, D. H. 2001, ApJ, 554, L55

Chung, S. M., Drake, J. J., Kashyap, V. L., Ratzlaff, P. W., \& Wargelin, B. J. 2004, in UV and Gamma-Ray Space Telescope Systems, ed. G. Hasinger, \& M. J. L. Turner, 5488, 51

Cohen, D. H. 2000, in The Be Phenomenon in Early-Type Stars, ed. M. A. Smith, H. F. Henrichs, \& J. Fabregat, IAU Colloq., 175, ASP Conf Ser., 214, 156

Cohen, D. H., Leutenegger, M. A., Grizzard, K. T., et al. 2006, MNRAS, 368, 1905
Cugier, H., \& Boratyn, D. A. 1992, Acta Astron., 42, 191

Daszyńska-Daszkiewicz, J., \& Niemczura, E. 2005, A\&A, 433, 1031 Donati, J.-F., Wade, G. A., Babel, J., et al. 2001, MNRAS, 326, 1265

Donati, J.-F., Babel, J., Harries, T. J., et al. 2002, MNRAS, 333, 55

Donati, J.-F., Howarth, I. D., Bouret, J.-C., et al. 2006, MNRAS, 365, L6

Drake, J. J., \& Testa, P. 2005, Nature, 436, 525

Dziembowski, W. A., \& Pamiatnykh, A. A. 1993, MNRAS, 262, 204

Gagné, M., Caillault, J.-P., Stauffer, J. R., \& Linsky, J. L. 1997, ApJ, 478, L87

Gagné, M., Oksala, M. E., Cohen, D. H., et al. 2005, ApJ, 628, 986

Gezari, D. Y., Labeyrie, A., \& Stachnik, R. V. 1972, ApJ, 173, L1

Grevesse, N., \& Sauval, A. J. 1998, Space Sci. Rev., 85, 161

Henrichs, H. F., de Jong, J. A., Donati, D.-F., et al. 2000, in Magnetic Fields of Chemically Peculiar and Related Stars, ed. Y. V. Glagolevskij, \& I. I. Romanyuk, 57

Henrichs, H. F., Schnerr, R. S., \& Ten Kulve, E. 2005, in The Nature and Evolution of Disks Around Hot Stars, ed. R. Ignace, \& K. G. Gayley, ASP Conf. Ser., 337, 114

Heynderickx, D., Waelkens, C., \& Smeyers, P. 1994, A\&AS, 105, 447

Hoogerwerf, R., Brickhouse, N. S., \& Mauche, C. W. 2004, ApJ, 610, 411

Hutchings, J. B., \& Hill, G. 1977, ApJ, 213, 111

Kashyap, V., \& Drake, J. J. 2002, in Stellar Coronae in the Chandra and XMMNEWTON Era, ed. F. Favata, \& J. J. Drake, ASP Conf. Ser., 277, 509

Kontizas, E., \& Theodossiou, E. 1980, MNRAS, 192, 745

Krelowski, J., \& Sneden, C. 1993, PASP, 105, 1141

Leutenegger, M. A., Paerels, F. B. S., Kahn, S. M., \& Cohen, D. H. 2006, ApJ, 650, 1096

Lucy, L. B., \& White, R. L. 1980, ApJ, 241, 300

Mewe, R., \& Schrijver, J. 1978, A\&A, 65, 99

Morel, T., Butler, K., Aerts, C., Neiner, C., \& Briquet, M. 2006, A\&A, 457, 651

Ness, J.-U., Brickhouse, N. S., Drake, J. J., \& Huenemoerder, D. P. 2003, ApJ, 598,1277

Niemczura, E., \& Daszyńska-Daszkiewicz, J. 2005, A\&A, 433, 659

Pan'ko, E. A., \& Tarasov, A. E. 1997, Astron. Lett., 23, 545

Pigulski, A., \& Boratyn, D. A. 1992, A\&A, 253, 178

Schnerr, R. S., Henrichs, H. F., Oudmaijer, R. D., \& Telting, J. H. 2006, A\&A, 459, L21

Schnerr, R. S., Henrichs, H. F., Owocki, S. P., Ud-Doula, A., \& Townsend, R. H. D. 2007, in Active OB-Stars: Laboratories for Stellare and Circumstellar Physics, ed. A. T. Okazaki, S. P. Owocki, \& S. Stefl, ASP Conf. Ser., 361, 488

Schulz, N. S., Canizares, C., Huenemoerder, D., \& Tibbets, K. 2003, ApJ, 595, 365

Schulz, N. S., Testa, P., Huenemoerder, D. P., Ishibashi, K., \& Canizares, C. R. 2006, ApJ, 653, 636

Stelzer, B., Flaccomio, E., Montmerle, T., et al. 2005, ApJS, 160, 557

Stibbs, D. W. N. 1950, MNRAS, 110, 395

Tian, B., Men, H., Deng, L.-C., Xiong, D.-R., \& Cao, H.-L. 2003, Chinese J. Astron. Astrophys., 3, 125

Townsend, R. H. D., \& Owocki, S. P. 2005, MNRAS, 357, 251

ud-Doula, A., \& Owocki, S. P. 2002, ApJ, 576, 413

Vaiana, G., Cassinelli, J., Fabbiano, G., et al. 1981, ApJ, 244, 163

Waldron, W. L., \& Cassinelli, J. P. 2007, ApJ, 668, 456

Zombeck, M. 1990, Handbook of Space Astronomy \& Astrophysics, 2nd edn. (Cambridge Univ. Press) 